\title{
Pricing and Hedging Options Conditional on Market Activity
}

\author{
Alec Kercheval1, Navid Salehy², Nima Salehy³ \\ ${ }^{1}$ Department of Mathematics, Florida State University, Tallahassee, FL, USA \\ ${ }^{2}$ Department of Mathematics, University of New Orleans, LA, USA \\ ${ }^{3}$ Department of Mathematics and Statistics, Louisiana Tech, Ruston, LA, USA \\ Email: akercheval@fsu.edu
}

How to cite this paper: Kercheval, A., Salehy, N. and Salehy, N. (2022) Pricing and Hedging Options Conditional on Market Activity. Journal of Mathematical Finance, 12, 1-19.

https://doi.org/10.4236/jmf.2022.121001

Received: October 4, 2021

Accepted: December 26, 2021

Published: December 29, 2021

Copyright (c) 2022 by author(s) and Scientific Research Publishing Inc. This work is licensed under the Creative Commons Attribution International License (CC BY 4.0).

http://creativecommons.org/licenses/by/4.0/

\begin{abstract}
We replicate and price European options on stocks modeled by time-changed geometric Brownian motion. The time change is obtained as the integrated intensity of random arrival times of price changes of the underlier over the life of the option. For European call options we obtain explicit hedging and pricing formulas. This approach is motivated by the need to connect option prices directly to the microstructure properties of the limit order book that determines tick-by-tick stock price changes. The continuous time model is obtained as an appropriate limit of discrete time random walks with random jump times, in the limit of infinitely many independent representative agents.
\end{abstract}

\section{Keywords}

Random Walks, Time-Changed Brownian Motion, Option Pricing, Limit Order Book

\section{Introduction}

In this paper we derive unique European option prices and hedging strategies in a Black-Scholes-style market for which the underlying stock process is timechanged by a measure of market activity. The time change is the compensator of an arrival process, and the option prices are conditional on the integrated intensity of the arrival process over the life of the option. In particular, options, such as call options, that depend only on the terminal price of the underlier may be perfectly replicated as a function of the terminal value of the compensator. In the case of European call options, we obtain an explicit hedging and price formulas as functions of the integrated intensity. 
We are motivated by efforts to model the dynamics of the limit order book that underlies stock price formation. From this perspective, stock price movements are the result of limit and market order arrivals collected in an electronic limit order book. Order arrival times are random and described by point processes, most notably by "self-exciting" Hawkes processes, e.g. [1]-[7]. It is these order arrivals that explain movements of the quoted market price. In this view, price heteroscedasticity is explained endogenously by the variability in arrival rates of orders to the order book, which determines the rate of price changes.

\subsection{The Model}

As a model for the high frequency limit order book microstructure, we consider price changes as the limit of generalized random walks for which the i.i.d. space increments (e.g. log price changes) occur at random times given by a simple point process and associated counting process. Considering the effect on market price to be the summed contribution of many small such changes, we obtain as a scaled limiting price process a time-changed Brownian motion, where the time change is the compensator of the original counting process. With mild assumptions, the resulting time-changed Brownian motion is a continuous square integrable martingale with uncorrelated, but not necessarily independent, increments, as is consistent with stylized facts about equity prices.

The limiting process serves as the basis for a Black-Scholes style option pricing model that we call "activity-driven Black-Scholes", to emphasize its connection to the market microstructure. It incorporates the characteristics of the chosen point process describing the "excitability" of order arrivals causing price changes in the underlying limit order book. This affords a perspective on a heteroscedastic stock price model for option pricing in which the fluctuating volatility can be intrinsically connected to a calibrated model of the underlying order book. When the point process is a homogeneous Poisson process with unit intensity, we recover the usual Brownian motion and Black-Scholes model.

When there is a second source of randomness-such as market activity-affecting the stock price, replication of an option in the ordinary Black-Scholes sense is not possible using only the underlying stock and a numeraire. But we can still obtain conditional pricing and replication. This is the content of our main theorem and corollaries in Section 4.

We mention two ways that this kind of conditional pricing and hedging can be useful. First, if there is an underlying calibrated limit order book model defining a market activity process, this will define a distribution of integrated intensities, and hence a calibrated distribution of options prices, distributed over the range of market activity outcomes. The width of this distribution is a way to measure the "volatility risk" of the option price. Second, the possible trajectories of market activity intensity can be considered a way to parametrize "scenarios" for use by banks in stress-testing options portfolios, as required by banking regulators. A single underlying market activity scenario $\Lambda(t)$ can be used to underly the prices of a basket of different options that share the same scenario. 


\subsection{Summary of Results}

We next briefly summarize our results. We say that a simple counting process $N$ is regular if the compensator $\Lambda$ of $N$ is continuous, strictly increasing, and $\Lambda(\infty)=\infty$. This is a mild assumption including most examples of interest.

For any regular counting process $N$ with compensator $\Lambda$ and for any integer $n \geq 1$ there is a natural regular counting process $N^{n}$ with compensator $\Lambda^{n}=n \Lambda$, which we use as follows. Given an i.i.d. sequence $\left\{\varepsilon_{i}: i \geq 1\right\}$ of random variables with $E\left[\varepsilon_{i}\right]=0, \operatorname{Var}\left(\varepsilon_{i}\right)=\sigma^{2}<\infty$, and independent of $N^{n}$ for all $n$, we define a sequence $\left\{\mathcal{S}_{N}^{n}: n=1,2,3, \cdots\right\}$ of rescaled random walks over $N$ by

$$
\mathcal{S}_{N}^{n}(t)=\frac{1}{\sqrt{n}} \sum_{i=1}^{N^{n}(t)} \varepsilon_{i}
$$

These random walks over $N$ can be compared to the standard sequence of rescaled random walks over deterministic (integer) times

$$
\mathcal{W}^{n}(t)=\frac{1}{\sqrt{n}} \sum_{i=1}^{\lfloor n t\rfloor} \varepsilon_{i},
$$

that are well-known (Donsker's Theorem [8]) to converge weakly in the Skorokhod topology to the process $\sigma \mathcal{B}$, where $\mathcal{B}$ is standard Brownian motion.

A similar fact is true for the random walks $\mathcal{S}_{N}^{n}$ over $N$ : the sequence $\left\{\mathcal{S}_{N}^{n}: n \geq 1\right\}$ of stochastic processes defined above converges weakly to the scaled, time-changed Brownian motion $\sigma \mathcal{B} \circ \Lambda$.

Since the time change $\Lambda$ need only be regular, we do not require it to be a Levy subordinator, or even Markov. This opens the door to "self-exciting" point processes such as those often used to model order arrivals in the high-frequency limit order book.

In general, $\mathcal{B}(\Lambda(t))$ is a continuous square integrable martingale; its quadratic variation is $\Lambda$; it is a standard Brownian motion if and only if $N$ is Poisson with unit intensity; it has uncorrelated increments, but independent increments if and only if $\Lambda$ is deterministic.

Our activity-driven Black-Scholes model for the stock price is

$$
S(t)=S_{0} \exp (\sigma \mathcal{B}(\Lambda(t))+\mu \Lambda(t)),
$$

where $\mathcal{B}$ is a standard Brownian motion, along with the numeraire

$$
B_{t}=\exp (r t) \text {. }
$$

This is flexible enough to display most of the stylized facts of stock price returns. For certain contingent claims $X$ paying off at the maturity time $T$, and conditional on the value of $\Lambda(T)$, we show $X$ is attainable (may be replicated by a self-financing strategy), find an equivalent martingale measure $Q$ for the discounted stock, and obtain a no-arbitrage pricing formula of the form

$$
V_{t}(X)=E_{Q}\left[\frac{B_{t}}{B_{T}} X \mid \mathcal{F}_{t}\right]
$$


This is stated in detail in Theorem 4, where we formalize conditioning on $\Lambda(T)$ by the condition that $\Lambda$ is "pinned" at $T$ (Section 3.2).

As an application, we can give explicit conditional hedging and pricing formulas (Corollary 3) for a vanilla European call option, conditional on the integrated intensity $\Lambda(T)=T^{\prime}$. The price formula at $t=0$ turns out to be the classical Black-Scholes formula, but with the strike price $K$ replaced by

$\mathrm{e}^{r\left(T^{\prime}-T\right)} K$, and the maturity $T$ replaced by $T^{\prime}$. The resulting call price is an increasing function of $T^{\prime}$, which can be considered a "market activity" or "market clock" parameter similar in effect to the volatility.

\subsection{Related Literature}

Financial applications of time-changed Lévy processes have been extensively studied, most significantly in the work of Peter Carr and collaborators, for example in [9] [10] [11] [12], and also others such as [13], and goes back to [14] and [15]. In most of this work the time change process and pricing measure are a priori ingredients in the model, whereas here we view the time change and pricing measure as derived features arising out of (a limit of) statistical behavior of traders, and we are focused on attainable claims.

Often in the literature attention is directed toward particular Markov time change processes such as integrated affine processes ([9] [12] [13]) due to the need to compute the Laplace transform of the time change. In [10], Carr and Lee can consider an arbitrary continuous time change process because the swap price they study is independent of the time change. In this paper we restrict attention to independent continuous time changes, but in a general setting in which they need not be Markov. We are particularly interested in time changes corresponding to self-exciting processes like the Hawkes process. (However, none of our results assume any process is Hawkes.)

The work of [16] is closely related to this paper. Although not explicitly considering time-changed processes, Bick values trading strategies depending on the "cumulative squared volatility" $[Y, Y]_{t}$ of the stock price $S_{t}$, where typically $Y_{t}=\log \left(S_{t} / S_{0}\right)$. In our activity-driven Black-Scholes model (1) this cumulative squared volatility is none other than a multiple $\sigma^{2}$ of our market activity parameter $\Lambda(t)$. Bick defines a dynamic trading strategy in $S_{t}$ and $B_{t}$ that depends on $[Y, Y]_{t}$ and which replicates a payoff of the form $h\left(S_{\tau}\right)$ to be paid at the stopping time $\tau$ defined by $[Y, Y]_{\tau}=q$, for a fixed threshold $q$. With proper restatement our corollaries 2 and 3 can be viewed as variations of Bick's results. However, neither the main proposition in [16] nor Theorem 4 implies the other. Bick's main result rests on Ito's Lemma and thus applies to general semi-martingale stock processes, while Theorem 4 is limited to time-changed Brownian motion. However, the latter is more general in the sense that it covers a larger class of payoffs, for example that might depend on the history of $S$. This level of generality significantly complicates the proof, which requires techniques beyond Ito's Lemma to confront the lack of a predictable representation property for time changed Brownian motion. See Section 6. 
Some extensions of [16] appear in [17], which focuses on the topic of model-free options on realized variance.

A related direction in the literature has been the study of option pricing for discrete time models motivated by the binomial tree model, e.g. [18] [19] [20]. In [21] the authors examine the weak convergence of discrete models where the jumps are general Bernoulli random variables, and study the corresponding convergence of option prices.

The comprehensive book [22] touches on this and also surveys a variety of papers examining various versions of binomial models with special forms of randomized time steps, such as [23] in which the time steps are derived from Poisson processes. Jacod and Shiryaev [24] develop some quite general convergence theorems that imply Donsker's theorem, but restrict attention to semimartingales with independent increments. The book [22] is primarily focused on the general question of whether option prices for discrete models converge to corresponding prices for the continuous-time weak limits. This is a subtle topic we have not addressed in this paper.

The remainder of the paper is organized as follows. In Section 2 we describe random walks over continuous time point processes and their rescalings and limits. Sections 3 and 4 describe our activity-driven Black-Scholes model, and the pricing and hedging of terminal-time-payoff options. It contains the main theorem, corollaries, and discussion. Section 5 summarizes, and the proof of the main theorem appears in Section 6.

\section{Random Walks for Many Agents}

\subsection{Discrete Time}

In this section we define some terms and describe a class of random walks over counting processes suited to our purposes. For background, see for example [22] [25] and [26].

We consider only simple, non-explosive point processes on $[0, \infty)$, i.e. sequences $\left\{T_{n}\right\}$ of $[0, \infty)$-valued random variables on a probability space $(\Omega, \mathbb{F}, P)$ such that $T_{0}=0, T_{n}<T_{n+1}$ a.s. for all $n$, and $\lim _{n \rightarrow \infty} T_{n}=+\infty$. For such a point process $\left\{T_{n}\right\}$, the corresponding counting process is

$$
N(t)=\sum_{n \geq 1} 1_{\left(T_{n} \leq t\right)}
$$

with $N(0)=0$. The natural filtration $\mathcal{F}_{t}^{N}=\sigma\left(N_{s}: s \leq t\right)$ of $N$ is automatically right continuous ([27], I.25). Assuming $E[N(t)]<\infty$ for all $t$, the DoobMeyer decomposition (e.g. [28]) gives us a unique, cadlag $\mathcal{F}^{N}$-predictable process $\Lambda$, the compensator of $N$, such that $\Lambda(0)=0$ a.s., $E[\Lambda(t)]<\infty$ for all $t$, and $N(t)-\Lambda(t)$ is a cadlag $\mathcal{F}^{N}$-martingale.

In this paper we restrict attention to the (large) class of regular counting processes as defined in the introduction.

To set notation, we denote the classical one-dimensional random walk $\mathcal{W}(t)$ to be a piecewise constant cadlag stochastic process defined by 


$$
\mathcal{W}(t)=\sum_{i=1}^{\lfloor t\rfloor} \varepsilon_{i}
$$

where $\left\{\varepsilon_{i}: i \geq 1\right\}$ is an i.i.d. sequence of random variables, and we assume $E\left[\varepsilon_{i}\right]=0$ and $\operatorname{Var}\left(\varepsilon_{i}\right)=\sigma^{2}<\infty$.

Motivated by the interpretation of $\mathcal{W}$ as a log-price process, we can think of $\mathcal{W}$ as the running sum of a sequence $\varepsilon_{i}$ of random contributions, or price shocks, delivered at times $i=1,2,3, \cdots$ by a representative agent at unit frequency as a result of trading.

Now instead of a single agent contributing at unit frequency, we can imagine $n$ smaller agents also contributing at unit frequency, but contributing only $\varepsilon_{i} / \sqrt{n}$ each independently, and where the deterministic contribution times are spread out uniformly, yielding

$$
\mathcal{W}^{n}(t)=\frac{1}{\sqrt{n}} \mathcal{W}(n t)=\frac{1}{\sqrt{n}} \sum_{i=1}^{\lfloor n t\rfloor} \varepsilon_{i} .
$$

This process converges weakly as $n \rightarrow \infty$ to the multiple $\sigma \mathcal{B}(t)$ of a standard Brownian motion $\mathcal{B}(t)$ (Donsker's Theorem). It can be interpreted, in the limit, as the running total of the contributions of infinitely many infinitesimal agents all contributing independently with unit frequency, and forms the basis of the Black-Scholes model.

Now we would like to imagine a different representative agent for which the contribution times are the random jump times of a regular counting process $N(t)$, instead of the deterministic times $t=1,2,3, \cdots$. The counting process may be quite general, including a Hawkes process or another self-exciting, nonMarkov process. We assume it is independent of the i.i.d. sequence $\left\{\varepsilon_{i}\right\}$. The corresponding random walk is denoted

$$
\mathcal{S}_{N}(t)=\mathcal{W}(N(t))=\sum_{i=1}^{N(t)} \varepsilon_{i} .
$$

If we denote by $\Lambda(t)$ the compensator of $N$, then $\Lambda$ may be interpreted as the time integral of an arrival rate intensity $\lambda(t)$, if $\Lambda$ is absolutely continuous with respect to $t$.

Corresponding to the scaling of the unit frequency agent, we may also imagine we have $n$ smaller agents, each contributing an independent amount $\varepsilon_{i} / \sqrt{n}$ with the same arrival intensity. The integrated arrival intensity of $n$ such agents will be $n \Lambda(t)$. It is straightforward to show that the corresponding counting process $N^{n}$ with compensator $n \Lambda$ may be defined by

$$
N^{n}(t)=N\left(\Lambda^{-1}(n \Lambda(t))\right) \text {. }
$$

(Here $\Lambda^{-1}(t)$ denotes the functional inverse of $\Lambda(t)$, well-defined since $\Lambda$ is strictly increasing.)

We may then define the scaled random walk defined by a regular counting process $N(t)$ to be

$$
\mathcal{S}_{N}^{n}(t)=\frac{1}{\sqrt{n}} \mathcal{W}\left(N^{n}(t)\right)=\frac{1}{\sqrt{n}} \sum_{i=1}^{N^{n}(t)} \varepsilon_{i} .
$$


As before, we can interpret this as the running total of the independent contributions of $n$ small agents, each with rate described by the compensator $\Lambda$ of $N$.

We note that, like the rescaled classical random walk, the random walk $\mathcal{S}_{N}^{n}$ is a martingale with respect to the natural filtration and there are explicit expressions for the variance and covariances in terms of $N$ [29].

\subsection{The Rescaled Limit of Infinitely Many Agents}

Just as the classical random walks $\mathcal{W}^{n}(t)$ converge weakly to $\sigma \mathcal{B}(t)$, so also the random walks $\mathcal{S}^{n}$ converge to a time-changed Brownian motion. A general reference on weak convergence in this context is [22], and a detailed treatment of the following theorem is given in [29].

Theorem 1 Let $\varepsilon_{i}$ be an i.i.d. sequence of random variables with mean 0 and variance $\sigma^{2}$. Given a regular counting process $N$ with compensator $\Lambda$, the sequence $\left\{\mathcal{S}_{N}^{n}: n \geq 1\right\}$ of rescaled random walks over $N$ converges weakly, in the Skorokhod metric, to the time-changed Brownian motion $\sigma \mathcal{B} \circ \Lambda$. Moreover, $\mathcal{B}$ and $\Lambda$ are independent.

The strategy of proof is to establish that $\left(\mathcal{W}^{n}, \frac{1}{n} N^{n}\right)$ converges weakly to $(\sigma \mathcal{B}, \Lambda)$ in the product space, and then apply the composition operator to both sides, using the Continuous Mapping Theorem and the continuity of composition.

Since we do not assume that $\Lambda$ is a subordinator, the limit $\sigma \mathcal{B} \circ \Lambda$, while continuous, need not be a Lévy process, or even Markov. Theorem 1 is, in spirit, a generalization of Donsker's theorem. The Donsker case is recovered when $N$ is chosen to be a homogeneous Poisson process with unit intensity, in which case the compensator is $\Lambda(t)=t$ and the weak limit becomes $\sigma \mathcal{B}(t)$, a multiple of standard Brownian motion.

The process $\mathcal{B} \circ \Lambda$ depends on $N$ because $\Lambda$ depends on $N$. To emphasize that dependence, we will use the notation

$$
\mathcal{B}^{N}=\mathcal{B} \circ \Lambda \text {, i.e. } \mathcal{B}^{N}(t)=\mathcal{B}(\Lambda(t)) \text {. }
$$

To put this into words:

The limiting random walk $\mathcal{B}^{N}$ is a time-changed Brownian motion, where the time change is the compensator of the point process describing the trading times of a representative infinitesimal agent.

Let $\left\{\mathcal{H}_{t}\right\}$ be the filtration defined by

$$
\mathcal{H}_{t}=\sigma\left(\mathcal{B}(r), \Lambda^{-1}(u): r \leq t, u \leq t\right) .
$$

By the usual augmentation if necessary, we assume that these filtrations satisfy the usual conditions.

It is shown in [29] that $\Lambda(t)$ is an $\mathcal{H}$-stopping time for each $t, \mathcal{B}^{N}$ is a continuous square integrable martingale with respect to the filtration $\mathcal{H}_{\Lambda}$, and $\left(\mathcal{B}^{N}\right)^{2}-\Lambda$ is a continuous martingale with respect to the same filtration.

Furthermore: 
1) $\left[\mathcal{B}^{N}, \mathcal{B}^{N}\right]_{t}=\Lambda(t)$,

2) $E\left[\mathcal{B}^{N}(t)\right]=0$,

3) $\operatorname{cov}\left(\mathcal{B}^{N}(s), \mathcal{B}^{N}(t)\right)=E[N(s \wedge t)]$ for all $s, t \geq 0$,

4) $\mathcal{B}^{N}$ has uncorrelated increments, and

5) $\mathcal{B}^{N}$ has independent increments if and only if $\Lambda$ is deterministic.

These properties make $\mathcal{B}^{N}$ useful as a model for financial price processes. Financial returns time series can have close to zero autocorrelation, but squared returns show positive autocorrelation due to typically observed heteroscedasticity and the corresponding failure of independence of returns. Choice of a nondeterministic $\Lambda$ naturally provides us with a stock price model consistent with these stylized facts of uncorrelated but dependent increments.

\section{Preliminary Topics}

Here we introduce some brief preliminaries prior to stating our main results.

\subsection{Hawkes Processes}

The Hawkes process (e.g. [1] [2] [4] [5] [30] among many references) is a popular counting process to model the arrival rates of orders to the limit order book, and hence the jump times of the stock price process. We may take $N$ to be a regular Hawkes process with intensity

$$
\lambda_{t}=\alpha+\beta \int_{0}^{t} \mu(t-s) N(\mathrm{~d} s)>0,
$$

where $\alpha>0$ and the response function $\mu(t)$ is a positive function satisfying $\int_{0}^{\infty} \mu(t) \mathrm{d} t<\infty$. The (non-Markov) compensator is $\Lambda(t)=\int_{0}^{t} \lambda_{t} \mathrm{~d} t$. Then the random walks $\mathcal{S}_{N}^{n}, n \geq 1$, can be the basis of a model for the log stock price that will reflect the self-exciting nature of the Hawkes process.

Modelers who use this approach to describe the activity of the high-frequency limit order book then have access to the option price model described below if they have a parametrized distribution for a terminal value $\Lambda(T)$.

\subsection{Pinned Processes}

Definition 2 A continuous random process $\Lambda(t)$ with $\Lambda(0)=0$ is pinned at time $T$ if $\Lambda(T)=T^{\prime}$ for some deterministic $T^{\prime}$.

The Brownian bridge is a familiar example. Absolutely continuous, increasing, even self-exciting pinned processes are not hard to come by. For example, let $r_{t}$ be any positive adapted RCLL process, such as the intensity of a Hawkes process. Let $R_{t}=\mathrm{e}^{-r_{t}}<1$. For any $T^{\prime}, c>0$ with $c<T^{\prime} / T^{2}$, define

$$
\Lambda(t)=\frac{T^{\prime}}{T} t-c(T-t) \int_{0}^{t} R_{s} \mathrm{~d} s .
$$

Then $\Lambda(0)=0, \Lambda(T)=T^{\prime}, \Lambda(\infty)=\infty, \Lambda$ is absolutely continuous, adapted to the natural filtration of $r$, and is increasing on $[0, \infty)$ because

$$
\Lambda^{\prime}(t)=\frac{T^{\prime}}{T}+c \int_{0}^{t} R_{s} \mathrm{~d} s-c(T-t) R_{t}>\frac{T^{\prime}}{T}-c(T-t)>0 .
$$


The following proposition demonstrates that regular counting processes with pinned compensators are easily constructed.

Proposition 3 Denote by $P(t)$ the homogeneous Poisson process with rate 1 , and let $\Lambda$ be any absolutely continuous, strictly increasing process pinned at time $T$. For each $t$, denote by $\mathcal{F}_{t}$ the sigma-algebra $\mathcal{F}_{t}^{P} \vee \mathcal{F}_{t}^{\Lambda^{-1}}=\sigma\left(P(s), \Lambda^{-1}(s): s \leq t\right)$. Then $\Lambda(t)$ is an $\mathcal{F}$-stopping time for each $t$, and

$$
N(t)=P(\Lambda(t))
$$

is a regular counting process on $[0, T]$ with compensator $\Lambda$ with respect to $\mathcal{F}_{\Lambda}$.

Proof. Given $s<t \leq T$, apply the optional stopping theorem (e.g. [31], II.3.2) to the $\mathcal{F}$-stopping times $\Lambda(s)$ and $\Lambda(t)$, bounded by $T^{\prime}=\Lambda(T)$, to the $\mathcal{F}$-martingale $P(t)-t$.

\subsection{Activity-Driven Black-Scholes}

The familiar Black-Scholes option pricing framework for a bond and stock price process $B_{t}$ and $S^{(B S)}(t)$, given by

$$
B_{t}=\exp (r t), \quad S^{(B S)}(t)=S_{0} \exp (\sigma \mathcal{B}(t)+\mu t),
$$

is often motivated as a limit of the exponential of a binomial random walk of the form $\mathcal{W}^{n}(t)$ discussed above. At short time scales, however, we tend to observe non-independent increments and some level of self-exciting behavior in the limit order book driving the price.

When $N$ is any regular point process describing the arrival of orders to the order book, we can call the corresponding limiting market model an "activity-driven Black-Scholes model", given by

$$
B_{t}=\exp (r t), \quad S(t)=S^{(B S)}(\Lambda(t))=S_{0} \exp \left(\sigma \mathcal{B}^{N}(t)+\mu \Lambda(t)\right),
$$

where $r, \sigma, \mu$ are positive constants. Our interpretation of the time change $\Lambda$ is the compensator of the arrival point process $N$ of trades for a representative infinitesimal agent in the market.

This turns out to be a suitable framework for option pricing that can incorporate heteroscedasticity and other non-stationary stylized facts of stock price behavior. The goal of this section is to show that in certain cases, conditional on $\Lambda(T)$, we can also obtain a no-arbitrage pricing formula analogous to the standard Black-Scholes formula.

\section{Main Results}

Recall the filtration $\mathcal{H}_{t}=\sigma\left(\mathcal{B}(r), \Lambda^{-1}(u): r, u \leq t\right)$ with respect to which $\Lambda(t)$ is a stopping time for each $t$. If we define

$$
\mathcal{G}_{t}=\mathcal{H}_{\Lambda_{t}},
$$

then both $\mathcal{B}^{N}$ and $\Lambda$, hence $S(t)$, are $\mathcal{G}$-adapted processes on $(\Omega, \mathbb{F}, P)$. 
It's convenient to define the additional filtrations, assumed complete:

$$
\begin{gathered}
\mathcal{F}_{t}^{B}=\sigma(\mathcal{B}(s): s \leq t) \subset \mathcal{H}_{t}, \\
\mathcal{F}_{t}^{\Lambda^{-1}}=\sigma\left(\Lambda^{-1}(s): s \leq t\right) \subset \mathcal{H}_{t}, \\
\mathcal{F}_{t}^{\Lambda}=\sigma(\Lambda(s): s \leq t), \\
\mathcal{F}_{t}^{S}=\sigma(S(s): s \leq t) \subset \mathcal{G}_{t} .
\end{gathered}
$$

To discuss the problem of option pricing, we review some standard terminology. We will say that a pair $\left(\phi_{t}, \psi_{t}\right)$ is a self-financing strategy if $\phi$ and $\psi$ are $\mathcal{G}$-predictable processes such that if $V_{t}=\phi_{t} S(t)+\psi_{t} B_{t}$, then $\mathrm{d} V_{t}=\phi_{t} \mathrm{~d} S(t)+\psi_{t} \mathrm{~d} B_{t}$. Here we interpret $\phi_{t}$ as the number of shares of stock held in a portfolio at time $t, \psi_{t}$ the same for the bond, and so $V_{t}$ is the time- $t$ portfolio value.

If we fix a deterministic maturity time $T$, we call a random payoff $X \in \mathcal{G}_{T}$ at time $T$ a claim. A claim $X$ is an attainable claim if there exists a self-financing strategy $(\phi, \psi)$ with

$$
X=V_{T}=\phi_{T} S(T)+\psi_{T} B_{T},
$$

i.e. the self-financing strategy replicates the claim at the terminal time. When this is the case, $(\phi, \psi)$ is a replicating portfolio for $X$, and $V_{t}$ will be the noarbitrage price of the claim $X$ at any time $t<T$.

In the classical Black-Scholes model with a stock and a bond, every claim is attainable (the market is complete) due to the martingale representation property for Brownian motion. In the context of the market model (2), a suitable representation property for the time-changed Brownian motion $\mathcal{B}^{N}$ is not available. Instead, we will show that certain classes of claims are attainable, and establish a familiar-looking conditional expectation formula for the claim price, with respect to a suitably defined measure. The remainder of this section spells out the details.

\section{Market Assumptions.}

1) $(\Omega, \mathbb{F}, P)$ is a probability space supporting a Brownian motion $\mathcal{B}(t)$ and an independent regular point process $N$ with absolutely continuous compensator $\Lambda$, with $\Lambda(t)=\int_{0}^{t} \lambda_{u} \mathrm{~d} u$, where $\lambda$ is left-continuous with right limits and for some $\varepsilon>0$, for all $u, \lambda_{u}>\varepsilon$ a.s.; and

2) there are two tradable assets, a stock $S(t)$ and bond $B_{t}$ given by

$$
B_{t}=\mathrm{e}^{r t}, \quad S(t)=S_{0} \exp \left(\sigma \mathcal{B}^{N}(t)+\mu \Lambda(t)\right), \quad \sigma, \mu>0, r \geq 0 .
$$

3) $X \in \mathcal{G}_{T} \cap L^{1}(\Omega, \mathbb{F}, P)$ is a European option contract payoff at a fixed maturity $T>0$.

Theorem 4 (Pricing and Hedging a European option) Under the Market Assumptions above, suppose further that the compensator $\Lambda$ of $N$ is pinned at $T$. Let $Q$ be the equivalent martingale measure defined by

$$
\frac{\mathrm{d} Q}{\mathrm{~d} P}=\exp \left(-\int_{0}^{\Lambda(T)} \gamma_{t} \mathrm{~d} \mathcal{B}(t)-\frac{1}{2} \int_{0}^{\Lambda(T)} \gamma_{t}^{2} \mathrm{~d} t\right)
$$


where

$$
\gamma_{t}=\frac{1}{\sigma}\left(\mu+\sigma^{2} / 2-\frac{r}{\lambda_{\Lambda^{-1}(t)}}\right), \quad 0 \leq t \leq \Lambda(T) .
$$

Then the discounted stock price $Z_{t}=B_{t}^{-1} S(t)$ (stopped at $T$ ) is a $(Q, \mathcal{G})$ -martingale, and $\tilde{\mathcal{B}}(t)=\mathcal{B}(t)+\int_{0}^{t} \gamma_{\mathrm{s}} \mathrm{d} s$ is a standard Brownian motion with respect to $(Q, \mathcal{H})$.

Moreover, suppose in addition that the payoff $X$ is $Q$-independent of $\mathcal{F}_{T}^{\Lambda}$.

Then

1) $X$ is attainable and its replicating portfolio is $(\phi, \psi)$, with value

$$
V_{t}=\phi_{t} S(t)+\psi_{t} B_{t} \text {, }
$$

where

$$
\phi_{t}=\eta_{\Lambda(t)}, \psi_{t}=\xi_{\Lambda(t)},
$$

and $(\eta, \xi)$ is the replicating portfolio for the payoff $X$ at time $\Lambda(T)$ in the standard risk-neutral Black-Scholes model with stock $Y(t)$ and bond $B_{t}$ given by

$$
B_{t}=\mathrm{e}^{r t}, \quad Y(t)=S_{0} \exp \left(\sigma \tilde{\mathcal{B}}(t)-\frac{1}{2} \sigma^{2} t\right), \quad t \leq \Lambda(T) .
$$

2) The unique no-arbitrage price $V_{t}$ of $X$ at time $t<T$ is

$$
V_{t}=\left(B_{t} / B_{T}\right) E_{Q}\left[X \mid \mathcal{G}_{t}\right] .
$$

The proof is postponed to the Appendix. We remark that the Theorem provides explicit hedging and pricing, conditional on $\Lambda(T)$, of a general class of European option payoffs that might potentially depend on the whole history of $S(t)$. Moreover, the hedging strategy is attainable at any time $t$ as long as $\Lambda(t)$ is observable at time $t$. The hypothesis that $\Lambda$ is pinned at $T$ is equivalent to pricing that is conditional on the value $\Lambda(T)$. The additional assumption that the payoff $X$ is $Q$-independent of $\mathcal{F}_{T}^{\Lambda}$ is made more concrete in the following Corollaries describing some interesting special cases.

Corollary 1 Under the Market Assumptions above, if $N$ is an inhomogeneous Poisson process with positive intensity, then $X$ is attainable and its unique noarbitrage price at time $t<T$ is

$$
V_{t}=\left(B_{t} / B_{T}\right) E_{Q}\left[X \mid \mathcal{G}_{t}\right],
$$

where the equivalent martingale measure is defined in Equation (4).

Proof. An inhomogeneous Poisson process has a deterministic intensity, hence deterministic compensator $\Lambda$. The conclusion is immediate from Theorem 4 .

Corollary 2 Under the Market Assumptions above, suppose that the compensator $\Lambda$ of the regular point process $N$ is pinned at time $T$. Let $X \in L^{1}$ be the payoff of a European option of the form $X=f(S(T))$ for some function $f$.

Then $X$ is attainable and the unique no-arbitrage price $V_{t}$ of $X$ at time $t<T$ is 


$$
V_{t}=\left(B_{t} / B_{T}\right) E_{Q}\left[X \mid \mathcal{G}_{t}\right],
$$

where the measure $Q$ is the equivalent martingale measure defined by Equation (4).

Moreover, the time 0 price of the option $X=f(S(T))$ is given by

$$
V_{0}=\mathrm{e}^{-r T} \int_{-\infty}^{\infty} f\left(S_{0} \mathrm{e}^{-\sigma^{2} T^{\prime} / 2+r T} \mathrm{e}^{\sigma y}\right) p\left(y ; \sigma^{2} T^{\prime}\right) \mathrm{d} y,
$$

where $T^{\prime}=\Lambda(T)$ and $p\left(y ; \sigma^{2} T^{\prime}\right)$ is the pdf of a normal distribution in $y$ with mean zero and variance $\sigma^{2} T^{\prime}$.

Proof of Corollary 2. Let $\Lambda(T)=T^{\prime}$. Since $S(T)=\exp (\sigma \mathcal{B}(\Lambda(T))+m \Lambda(T))=\exp \left(\sigma \mathcal{B}\left(T^{\prime}\right)+m T^{\prime}\right)$, it follows that $S(T)$, hence $X$, is independent of $\Lambda$ and $\Lambda^{-1}$. The first part then follows from Theorem 4.

The second part follows straightforwardly from (8) and the fact, as shown in the proof of Theorem 4 , that

$$
\begin{aligned}
S(T) & =S_{0} \exp \left(\sigma \tilde{\mathcal{B}}(\Lambda(T))-(1 / 2) \sigma^{2} \Lambda(T)+r T\right) \\
& =S_{0} \exp \left(\sigma \tilde{\mathcal{B}}\left(T^{\prime}\right)-(1 / 2) \sigma^{2} T^{\prime}+r T\right)
\end{aligned}
$$

where $\tilde{\mathcal{B}}\left(T^{\prime}\right)$ is normally distributed with respect to $Q$ with mean zero and variance $T^{\prime}$.

Corollary 3 Under the same assumptions of Corollary 2, if $X=\left(S_{T}-K\right)^{+}$is a European call option with strike $K$ and maturity $T$, then its time- 0 price is

$$
V_{0}=C_{B S}\left(\sigma, r, S_{0}, \mathrm{e}^{r\left(T^{\prime}-T\right)} K, T^{\prime}\right),
$$

where $T^{\prime}=\Lambda(T)$ and $C_{B S}\left(\sigma, r, S_{0}, K, T\right)$ denotes the standard Black-Scholes call price at strike $K$ and maturity $T$.

Furthermore, the hedging portfolio $\left(\phi_{t}, \psi_{t}\right)$ is given explicitly by

$$
\phi_{t}=N\left(d_{1}^{\Lambda}(t)\right) \text { and } \psi_{t}=-K \mathrm{e}^{-r T^{\prime}} N\left(d_{2}^{\Lambda}(t)\right),
$$

where $N$ is the standard normal cumulative distribution function, and

$$
\begin{gathered}
d_{1}^{\Lambda}(t)=\frac{1}{\sigma \sqrt{T^{\prime}-\Lambda(t)}}\left[\ln \left(\frac{S_{t}}{K}+\left(r+\sigma^{2} / 2\right)\left(T^{\prime}-\Lambda(t)\right)\right)\right], \\
d_{2}^{\Lambda}(t)=d_{1}^{\Lambda}(t)-\sigma \sqrt{T^{\prime}-\Lambda(t)},
\end{gathered}
$$

and the time- $t$ price of the option is

$$
V_{t}=\phi_{t} S_{t}+\psi_{t} \mathrm{e}^{r t}
$$

Corollary 3 follows from Corollary 2, part (a) of Theorem 4, and the standard Black-Scholes formulas for the replicating portfolio $(\eta, \xi)$ of a European call in the market (6).

Notice that in the above results the quantity $\Lambda(t)$ is observable at time $t$, as it is the quadratic variation of $(\log S) / \sigma$ up to time $t$. Therefore the price formulas and replicating portfolios depend only on the random variable $T^{\prime}=\Lambda(T)$. We can view Equations (9) and (10) as the price conditional on 
$\Lambda(T)$, and therefore a scenario-based price, where the scenarios are parametrized by $\Lambda(T)$. These results would be relevant to stress-testing portfolio values under different possible future regimes of accumulated market activity over the life of the option.

Alternatively, if we have a separately calibrated limit order book model with arrival process $N$ leading to a parametrized distribution for $\Lambda(T)$, we can take a further expectation of Equation (10) to obtain an unconditional option price.

It is straightforward to check that the expression in Equation (10) is increasing in $T^{\prime}=\Lambda(T)$ when the other parameters are held constant. Therefore one can think of the accumulated intensity (or realized quadratic variation) $\Lambda(T)$ over the life of the option as playing a similar role as the volatility parameter $\sigma$ in affecting the option price. The parameter $\Lambda(T)$ is a "trading activity" parameter separate from volatility but having a similar qualitative effect on the option price. This model is therefore an alternative to a stochastic volatility model.

For a numerical illustration comparing Black-Scholes volatility $\sigma$ as a parameter to the integrated arrival intensity $\Lambda(T)$, see Figure 1. There we compare the effect of varying $\Lambda(T)$ in the activity-driven model to the effect of varying volatility $\sigma$ in the standard Black-Scholes model. We can see that $\Lambda(T)$ has characteristics similar to the Black-Scholes $\sigma$, and therefore is a reasonable alternative (random) measure of overall volatility.

In Figure 2, we look at how implied volatility depends on $\Lambda(T)$ for a fixed set of parameters. We also compare that to the implied volatilities referenced to a fixed maturity $T=0.2$ (years), for ordinary Black-Scholes call prices computed for variable $T$ as a proxy. Here we observe that $\Lambda(T)$ is non-linearly related to implied volatility, and similar to, but not captured by, varying the maturity parameter $T$ in the Black-Scholes formula.
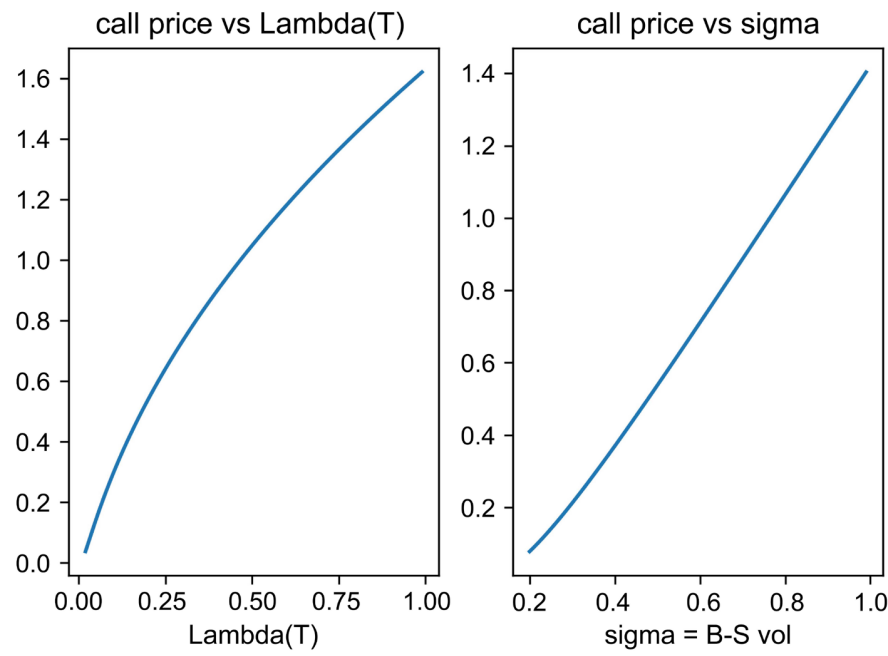

Figure 1. Call price comparison. On the left is the call price in the activity-driven model as a function of $\Lambda(T)$, for parameters stock price $S=10$, interest rate $r=0.03$, volatility $\sigma=0.5$, strike $K=11$, and maturity $T=0.2$. On the right is the standard BlackScholes call price for these parameters, but variable $\sigma$. 


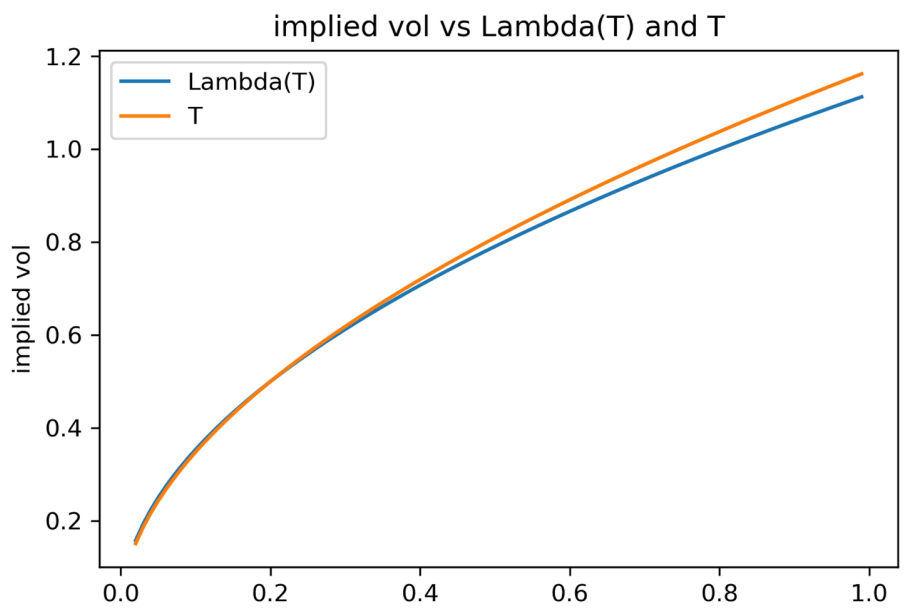

Figure 2. Black-Scholes implied volatility as a function of the maturity parameter $T$, and in the activity-driven model as a function of $\Lambda(T)$. The two should agree when $\Lambda(T)=T=0.2$. The parameters are stock price $S=10$, interest rate $r=0.03$, volatility $\sigma=0.5$, strike $K=11$, and base maturity $T=0.2$.

\section{Conclusions}

Motivated by the random jump times in the limit order book for a stock price and Theorem 1, we consider a generalized Black-Scholes model with a bond or cash account $B_{t}=\mathrm{e}^{r t}$ and a stock $S(t)=S_{0} \exp \left(\sigma \mathcal{B}^{N}(t)+\mu \Lambda(t)\right)$. This market can be thought of as a continuous limit of a discrete stock price model where price changes are driven by the action of many small agents acting with integrated intensity $\Lambda$, reflecting underlying limit order book activity. In this sense the market model's heteroskedastic features are derived from market-clock variations rather than imposed by an exogenously estimated stochastic volatility.

For certain classes of option payoffs $X$, and conditional on $\Lambda(T)$, we establish an option pricing formula in a familiar form

$$
V_{t}=\left(B_{t} / B_{T}\right) E_{Q}\left[X \mid \mathcal{H}_{\Lambda(t)}\right] \text {, }
$$

where $Q$ is the explicitly defined risk-neutral measure and $\mathcal{H}=\mathcal{F}^{\mathcal{B}} \vee \mathcal{F}^{\Lambda^{-1}}$. As an application, we can price a European call option with strike $K$ and maturity $T$, conditional on the value $\Lambda(T)=T^{\prime}$ of the integrated intensity of the counting process $N$ over the life of the option, as

$$
C_{B S}\left(\sigma, r, S_{0}, \mathrm{e}^{r\left(T^{\prime}-T\right)} K, T^{\prime}\right),
$$

where $C_{B S}$ is the usual Black-Scholes call option price formula. A separately calibrated risk-neutral distribution of $\Lambda(T)$ could provide an unconditional call option price as a function of parameters of a limit order book model.

\section{Proof of Theorem 4}

Given $X$, our goal is to construct a self-financing strategy in the stock and bond with time- $t$ value given by $V_{t}$ of Equation (7). 
In this proof we use the convention that when we call a process $M(t)$ a martingale that is only defined on an interval $\left[0, t_{0}\right]$, we mean that the process stopped at $t_{0}$ is a martingale.

Since $\Lambda$ is absolutely continuous with derivative $\lambda$ bounded below by $\varepsilon>0$, the inverse function theorem tells us that

$$
\Lambda^{-1}(t)=\int_{0}^{t} \bar{\lambda}_{u} \mathrm{~d} u,
$$

where

$$
\bar{\lambda}_{u}=\frac{1}{\lambda_{\Lambda^{-1}(u)}}
$$

is positive and bounded above by $1 / \varepsilon$. It follows that

$$
\gamma_{t}=\frac{1}{\sigma}\left(\mu+\sigma^{2} / 2-r \overline{\lambda_{t}}\right)
$$

is bounded and left continuous with right limits. By Girsanov's Theorem ([27], theorem III.42), the equivalent measure $Q$ on $(\Omega, \mathbb{F})$ defined by Equation (4) is such that, for $0 \leq t \leq \Lambda(T)$,

$$
\begin{aligned}
\tilde{\mathcal{B}}(t) & =\mathcal{B}(t)+\int_{0}^{t} \gamma_{s} \mathrm{~d} s \\
& =\mathcal{B}(t)+\frac{\mu+\sigma^{2} / 2}{\sigma} t-\frac{r}{\sigma} \int_{0}^{t} \bar{\lambda}_{u} \mathrm{~d} u \\
& =\mathcal{B}(t)+\frac{\mu+\sigma^{2} / 2}{\sigma} t-\frac{r}{\sigma} \Lambda^{-1}(t)
\end{aligned}
$$

is a standard Brownian motion with respect to $(Q, \mathcal{H})$. Following our convention, we write

$$
\tilde{\mathcal{B}}^{N}(t)=\tilde{\mathcal{B}}(\Lambda(t))=\mathcal{B}^{N}(t)+\frac{\mu+\sigma^{2} / 2}{\sigma} \Lambda(t)-\frac{r}{\sigma} t .
$$

$\tilde{\mathcal{B}}^{N}(t)$ is a square integrable $(Q, \mathcal{G})$-martingale, and since $\left[\tilde{\mathcal{B}}^{N}, \tilde{\mathcal{B}}^{N}\right]=\Lambda$, therefore so is the discounted stock price

$$
Z_{t} \equiv B_{t}^{-1} S(t)=S_{0} \exp \left(\sigma \mathcal{B}^{N}(t)+\mu \Lambda(t)-r t\right)=S_{0} \exp \left(\sigma \tilde{\mathcal{B}}^{N}(t)-\frac{1}{2} \sigma^{2} \Lambda(t)\right) .
$$

We are assuming that the option payoff $X$ is independent of $\mathcal{F}_{T}^{\Lambda}$. This is equivalent to the independence of $X$ from $\mathcal{F}_{\Lambda(T)}^{\Lambda^{-1}}$, since these two sigma-algebras are equal.

To construct a self-financing replicating portfolio, the difficulty is that the Predictable Representation Property (PRP, [31], V.4) enjoyed by Brownian motion does not necessarily hold for arbitrary continuous square integrable martingales like $\mathcal{B}^{N}$. The PRP for Brownian motion is what makes the BlackScholes option pricing theory work.

Our strategy is to apply the PRP to the Brownian motion model, and then change variables by means of the time change $\Lambda$ and the optional stopping theorem.

Recall that $\mathcal{B}^{N}, \Lambda$, and $S$ are adapted to the filtration $\mathcal{G}_{t}=\mathcal{H}_{\Lambda_{t}}$, and $\mathcal{B}$, 
$\Lambda^{-1}$ are adapted to $\mathcal{H}_{t}$.

Let

$$
Y(t)=Z\left(\Lambda^{-1}(t)\right)=S_{0} \exp \left(\sigma \mathcal{B}(t)-\frac{1}{2} \sigma^{2} t\right) .
$$

Evidently $Y$ is a $\left(Q, \mathcal{F}^{\tilde{\mathcal{B}}}\right)$ martingale.

Now define

$$
E(t)=E_{Q}\left[B_{T}^{-1} X \mid \mathcal{H}_{t}\right]
$$

Since

$$
\mathcal{H}=\mathcal{F}^{\mathcal{B}} \vee \mathcal{F}^{\Lambda^{-1}}=\mathcal{F}^{\tilde{\mathcal{B}}} \vee \mathcal{F}^{\Lambda^{-1}},
$$

the independence of $X$ from $\mathcal{F}_{\Lambda(T)}^{\Lambda^{-1}}$ implies $X \in \mathcal{F}_{\Lambda(T)}^{\tilde{\mathcal{B}}}$ and

$$
E(t)=E_{Q}\left[B_{T}^{-1} X \mid \mathcal{F}_{t}^{\tilde{\mathcal{B}}}\right] \text { for } t \leq \Lambda(T) \text {. }
$$

Since $E_{Q}\left[B_{T}^{-1} X \mid \mathcal{F}_{t}^{\tilde{\mathcal{B}}}\right]$ is a $\left(Q, \mathcal{F}^{\tilde{\mathcal{B}}}\right)$-martingale, by the Brownian martingale representation property (e.g. [31], V.3), there is an $\mathcal{F}^{\tilde{\mathcal{B}}}$-predictable, hence $\mathcal{H}$ -predictable, process $\eta_{t}$ such that

$$
\mathrm{d} E(t)=\eta_{t} \mathrm{~d} Y(t) .
$$

Next we wish to compose this equation with $\Lambda$, which is justified by the following lemma.

Lemma 5 ([32], lemma 2.3) Let $\mathcal{H}$ be a filtration satisfying the usual conditions and $X$ be an $\mathcal{H}$-semimartingale that is $C$-continuous, where $C$ is a finite $\mathcal{H}$-time-change. Let $L(X, \mathcal{H})$ denote the class of $\mathcal{H}$-predictable processes $\mathcal{H}$ for which the stochastic integral $\int_{0}^{t} H_{s} \mathrm{~d} X_{s}$ can be constructed.

If $H \in L(X, \mathcal{H})$, then $H_{C_{t^{-}}} \in L\left(X_{C}, \mathcal{H}_{C}\right)$ for all $t$. Moreover, with probability one, for all $t \geq 0$,

$$
\int_{0}^{C_{t}} H_{s} \mathrm{~d} X_{s}=\int_{0}^{t} H_{C_{s^{-}}} \mathrm{d} X_{C_{s}} .
$$

Since $\Lambda$ is a finite time-change with respect to $\mathcal{H}$, and letting $\phi_{t}=\eta_{\Lambda(t)}$, then $\phi_{t}$ is $\mathcal{H}_{\Lambda}$-predictable and we obtain from Equation (14) that

$$
\mathrm{d} E(\Lambda(t))=\phi_{t} \mathrm{~d} Z(t) .
$$

We may now consider a portfolio holding $\phi_{t}$ shares of stock and $\psi_{t}=E(\Lambda(t))-\phi_{t} Z(t)$ shares of the bond at time $t$.

The portfolio value process

$$
V_{t}=\phi_{t} S(t)+\psi_{t} B_{t}=B_{t} E(\Lambda(t))
$$

is self-financing by virtue of an easy computation using (15).

To complete the argument, the optional stopping theorem (e.g. [31], II.3) tells us that for any $t \in[0, T]$ :

$$
E(\Lambda(t))=E_{Q}\left[B_{T}^{-1} X \mid \mathcal{H}_{\Lambda(t)}\right] .
$$

Our portfolio strategy is therefore a replicating strategy because 


$$
V_{T}=B_{T} E(\Lambda(T))=B_{T} E_{Q}\left[B_{T}^{-1} X \mid \mathcal{H}_{\Lambda(T)}\right]=X .
$$

Therefore the no-arbitrage price of $X$ at any earlier time $t$ must be the value of the replicating portfolio

$$
V_{t}=B_{t} E(\Lambda(t))=\left(B_{t} / B_{T}\right) E_{Q}\left[X \mid \mathcal{H}_{\Lambda(t)}\right]
$$

as desired. This completes the proof of Theorem 4 .

\section{Acknowledgements}

The first author was partially supported by a Simons Collaboration Grant for Mathematicians.

\section{Conflicts of Interest}

The authors declare no conflicts of interest regarding the publication of this paper.

\section{References}

[1] Hawkes, A.G. (1971) Spectra of Some Self-Exciting and Mutually Exciting Point Processes. Biometrika, 58, 83-90. https://doi.org/10.1093/biomet/58.1.83

[2] Abergel, F. and Jedidi, A. (2015) Long-Time Behavior of a Hawkes Process-Based Limit Order Book. SIAM Journal on Financial Mathematics, 6, 1026-1043. https://doi.org/10.1137/15M1011469

[3] Bacry, E., Mastromatteo, I. and Muzy, J.-F. (2015) Hawkes Processes in Finance. Market Microstructure and Liquidity, 1, Article No. 1550005. https://doi.org/10.1142/S2382626615500057

[4] Zhu, L. (2014) Limit Theorems for a Cox-Ingersoll-Ross Process with Hawkes Jumps. Journal of Applied Probability, 51, 699-712. https://doi.org/10.1239/jap/1409932668

[5] Embrechts, P., Liniger, T. and Lin, L. (2011) Multivariate Hawkes Processes: An Application to Financial Data. Journal of Applied Probability, 48, 367-378. https://doi.org/10.1017/S0021900200099344

[6] Toke, I.M. (2011) "Market Making" in an Order Book Model and Its Impact on the Spread. In: Abergel, F., Chakrabarti, B.K., Chakraborti, A. and Mitra, M., Eds., Econophysics of Order-Driven Markets, Springer, Milano, 49-64. https://doi.org/10.1007/978-88-470-1766-5 4

[7] Errais, E., Giesecke, K. and Goldberg, L. (2010) Affine Point Processes and Portfolio Credit Risk. SIAM Journal on Financial Mathematics, 1, 642-665. https://doi.org/10.1137/090771272

[8] Billingsley, P. (1968) Convergence of Probability Measures. John Wiley and Sons, New York.

[9] Carr, P., Geman, H., Madan, D. and Yor, M. (2003) Stochastic Volatility for Levy Processes. Mathematical Finance, 13, 345-382. https://doi.org/10.1111/1467-9965.00020

[10] Carr, P. and Lee, R. (2013) Variation and Share-Weighted Variation Swaps on TimeChanged Lévy Processes. Finance and Stochastics, 17, 685-716. https://doi.org/10.1007/s00780-013-0212-9 
[11] Carr, P., Lee, R. and Wu, L. (2012) Variance Swaps on Time-Changed Levy Processes. Finance and Stochastics, 16, 335-355. https://doi.org/10.1007/s00780-011-0157-9

[12] Carr, P. and Wu, L. (2004) Time-Changed Levy Processes and Option Pricing. Journal of Financial Economics, 71, 113-141. https://doi.org/10.1016/S0304-405X(03)00171-5

[13] Hieber, P. and Scherer, M. (2012) A Note on First Passage Times of Continuously Time-Changed Brownian Motion. Statistics and Probability Letters, 82, 165-172. https://doi.org/10.1016/j.spl.2011.09.018

[14] Clark, P.K. (1973) Subordinated Stochastic Process Model with Finite Variance for Speculative Prices. Econometrica, 41, 135-155. https://doi.org/10.2307/1913889

[15] Mandelbrot, B. and Taylor, H.M. (1967) On the Distribution of Stock Price Differences. Operations Research, 15, 1057-1062. https://doi.org/10.1287/opre.15.6.1057

[16] Bick, A. (1995) Quadratic-Variation-Based Dynamic Strategies. Management Science, 41, 722-732. https://doi.org/10.1287/mnsc.41.4.722

[17] Carr, P. and Lee, R. (2010) Hedging Variance Options on Continuous Semi-Martingales. Finance and Stochastics, 14, 179-207. https://doi.org/10.1007/s00780-009-0110-3

[18] Cox, J.C., Ross, S.A. and Rubinstein, M. (1979) Option Pricing: A Simplified Approach. Journal of Financial Economics, 7, 229-263. https://doi.org/10.1016/0304-405X(79)90015-1

[19] Madan, D., Milne, F. and Shefrin, H. (1989) The Multinomial Option Pricing Model and Its Brownian and Poisson Limits. Review of Financial Studies, 2, 251-265. https://doi.org/10.1093/rfs/2.2.251

[20] Remillard, B., Hocquard, A., Lamarre, H. and Papageorgiou, N. (2017) Option Pricing and Hedging for Discrete Time Regime-Switching Models. Modern Economy, 8, 1005-1032. https://doi.org/10.4236/me.2017.88070

[21] Rachev, S.T. and Ruschendorf, L. (1995) Models for Option Prices. Theory of Probability \& Its Applications, 39, 120-152. https://doi.org/10.1137/1139005

[22] Prigent, J.-L. (2003) Weak Convergence of Financial Markets. Springer-Verlag, Berlin. https://doi.org/10.1007/978-3-540-24831-6

[23] Dengler, H. and Jarrow, R.A. (1997) Option Pricing Using a Binomial Model with Random Time Steps (A Formal Model of Gamma Hedging). Review of Derivatives Research, 1, 107-138. https://doi.org/10.1007/BF01531595

[24] Jacod, J. and Shiryaev, A.N. (1987) Limit Theorems for Stochastic Processes. Vol. 288, Springer-Verlag, Berlin. https://doi.org/10.1007/978-3-662-02514-7

[25] Hautsch, N. (2004) Modeling Irregularly Spaced Financial Data: Theory and Practice of Dynamic Duration Models. Springer-Verlag, Berlin. https://doi.org/10.1007/978-3-642-17015-7

[26] Whitt, W. (2002) Stochastic-Process Limits. Springer-Verlag, New York. https://doi.org/10.1007/b97479

[27] Protter, P. (2005) Stochastic Integration and Differential Equations. Springer-Verlag, Berlin. https://doi.org/10.1007/978-3-662-10061-5

[28] Fleming, T. and Harrington, D. (1991) Counting Processes and Survival Analysis. Wiley, Hoboken.

[29] Salehy, S.N. (2019) A New Price Model and a Black-Scholes Analog through Limits of Random Walks over Point Processes. PhD Thesis, Florida State University, Tallahassee. 
[30] Abergel, F., Anane, M., Chakraborti, A., Kedidi, A. and Muni Toke, I. (2016) Limit Order Books. Cambridge University Press, Cambridge. https://doi.org/10.1017/CBO9781316683040

[31] Revuz, D. and Yor, M. (1991) Continuous Martingales and Brownian Motion. Vol. 293, Springer-Verlag, Berlin. https://doi.org/10.1007/978-3-662-21726-9

[32] Kobayashi, K. (2011) Stochastic Calculus for a Time-Changed Semimartingale and the Associated Stochastic Differential Equations. Journal of Theoretical Probability, 24, 789-820. https://doi.org/10.1007/s10959-010-0320-9 